\title{
AUTOBIOGRAFIA PROFESIONAL UNA VÍA PARA LA AUTOFORMACIÓN DOCENTE
}

Laura J. Garcia R*

\section{La autobiografia: modalidad eflexión cadora}

Considerar a cada docente como constructor de su propia línea profesional, le otorga gran relevancia a sus narraciones autobiográficas por su carácter crítico y comprensivo, y al mismo tiempo constituyen un aporte a los modelos de formación del docente, que deben responder con mayor impacto a las demandas y exigencias de los mismos profesionales de la docencia y en forma creativa e innovadora, propiciar la reflexión y comprensión de los componentes pedagógicos, posibilitando la superación permanente del profesorado.

Varios investigadores como HUBER (1988), MEDINA (1988), MEDINA Y DOMÍNGUEZ $(1989,1991,1995)$ han constatado la importancia y el recurso que representa el análisis autobiográfico, al permitir que el docente profundice en su conocimiento personal y profesional, se pretende hacer historia, la historia profesional. Mediante la indagación crítica y la retrospección de los acontecimientos ocurridos en el aula y en los centros educativos, se realiza un estudio organizado, y riguroso para encontrar un modelo de autoformación del profesorado. Las investigaciones al respecto apoyan que la autobiografía es un paso previo a una acción formativa con docentes. Medina (1995) resalta que la autobiografía es más que una metodología y le permite al docente:

- Capacitarse para conocer las bases de su práctica educativa.

- Profundizar en las razones por las que decidió ser docente. 
- Consolidar su autoconcepto desde una vertiente positiva. profesional

- Seleccionar y comprender las experiencias en su realización

- Aprovechar su experiencia como base de su formación futura.

- Constatar su línea de desarrollo profesional con la de otros cole-

gas.

- Poner en tela de juicio su trayectoria profesional.

La autobiografía es un medio que permite al docente, tomar conciencia de la realidad cotidiana, y comprender lo complejo de su actuación, enmarcada dentro de su modelo didáctico, que se apoya en sus creencias; concepciones epistemológicas e ideológicas, concepciones sobre educación y enseñanza, que guía toda su práctica educativa. Así como también, le facilita al docente visualizar su evolución personal y profesional, es un esfuerzo continuo de profundización de lo realizado, un estudio para identificar y resolver problemas pedagógicos, al establecer un seguimiento para entenderlos en toda su complejidad. Por último, propicia también el desarrollo de los niveles descriptivos, analíticos, explicativos y valorativos del proceso de investigación y reflexión de su actuación docente, pues la reflexión sistemática, bien estructurada, y bajo una perspectiva de continuo, cada paso apoyada en el anterior, permite consolidar un saber práctico de gran proyección y trascendencia profesional.

Es necesario puntualizar algunos aspectos que esta metodología lleva implícito, reflexionar sobre la experiencia profesional, no basta para identificar los puntos claves de la línea profesional, es fundamental el razonamiento que apoya la acción, es necesaria la especulación teórica que se realice al respecto y la contextualización del hecho educativo, en referencia a otros docentes, a la institución educativa. Además, la valoración de la acción y su significación, es un elemento clave para su posterior consideración en el modelo que se quiere conformar.

El análisis autobiográfico, encuentra mayor proyección al someterse al estudio interbiográfico, e identificar puntos de coincidencia y sus claves de explicación compartidas, con otras biografías, el contraste enriquece el modelo de autodesenvolvimiento profesional y permite mayor comprensión y significación a las autobiografías.

\section{Metodologia de investigación aplicada al análisis autobio gráfico}

La metodología cualitativa en forma general se refiere a las investigaciones que producen datos descriptivos, donde el escenario donde se desarrollan los acontecimientos y las personas involucradas, tienen que ser 
observadas, comprendidas y analizadas desde una perspectiva holística, dentro del marco de referencia de ellas mismas. La perspectiva fenomenológica es esencial para comprender la metodología cualitativa, por lo tanto es importante experimentar la realidad desde el punto de vista de otras personas

Dentro de la perspectiva fenomenológica, se puede realizar un estudio centrándose dentro de dos enfoques teóricos principales, el interaccionismo simbólico y la etnometodología.

El interaccionismo simbólico, tal como lo expresa Taylor y Bogdan (1987) atribuye una importancia primordial a los significados sociales que las personas asignan al mundo que las rodea, la cultura de los grupos humanos le otorga diversos significados a una forma de conducta. Haciendo referencia al plano pedagógico, los docentes atribuyen significados partiendo desde sus propias concepciones y creencias que están impregnadas de gran significado social.

Taylor y Bogdan (1987) se refieren a la etnometodología como la teoría que fundamenta el significado de las acciones, a la aplicación que las personas hacen de reglas culturales abstractas y percepciones de sentido común a situaciones concretas, para que las acciones parezcan rutinarias, explicables y carentes de ambigüedad. Por lo tanto, los significados son un logro práctico por parte de los miembros de la sociedad. En el campo educativo este enfoque agudiza los estudios, pues los convierte en más complejos y multivariados.

La cita de estos diversos enfoques teóricos, tienen implicación directa cuando se esta planteando la autobiografía, dentro de una visión cualitativa, y como una perspectiva de investigación, con el propósito de configurar teorías o modelos de autodesenvolvimiento profesional, son puntos de partida para el análisis autobiográfico y concretar la metodología en este campo, aunque no se puede renunciar a la posibilidad de emplear una metodología cuantitativa cuando las condiciones estén dadas. Son diversas las metodologías que pueden ser empleadas para profundizar el auto análisis biográfico, entre ellas, la metodología clínica tiene gran pertinencia, pues permite indagar mediante interrogantes en la realidad personal, a objeto de identificar puntos claves en la línea profesional que sirvan de orientación creadora y que argumenten un modelo de autoformación. Medina (1996) destaca lo complementario que resulta a la metodología clínica la introspección, resalta la importancia de descubrir el significado riguroso de la experiencia formativa, y el mismo docente es el que valora la interdependencia de los datos.

La autobiografía necesita una metodología propia para elaborar desde ella una teoría o modelo de enseñanza. Woods (1987) define los modelos en los análisis cualitativos como la representación en una escala reducida de los componentes esenciales de procesos muy complicados, susceptibles de una rápida captación por nuestros limitados intelectos. Para el análisis autobiográfico, es necesario apoyarse en teorías que sirvan de base para la narración 
y discriminación de experiencias más formativas, pero también entran en juego la creación y reformulación de otras teorías. Esta dialéctica hace progresar la ciencia y brinda nuevas explicaciones, más profundas, más variadas de la realidad, la teoría se va construyendo progresivamente.

El modelo que se pretende conformar surge de la reflexión profunda en la acción, puntualizando algunos aspectos de la enseñanza, aportando las concepciones, creencias y argumentos por los cuales realiza la enseñanza tal como la aplica.

En el proceso de análisis de datos de la autobiografía, puede partir del conocimiento explorativo y dimensión significativa que encierra la información, el eje del proceso pude ser cronológico si es la historia la que encierra un mayor nivel de significación o sincrónico si existen hechos con carácter determinante para conocer lo que esta sucediendo realmente, luego se esquematiza el modelo surgido para facilitar la comprensión del mismo.

El establecimiento de modelos, lleva consigo una serie de pasos previos, se pasa de la descripción a la interpretación y la teoría a través de conceptos y proposiciones, los conceptos se utilizan para iluminar los procesos y los fenómenos sociales que no son fácilmente perceptibles en las descripciones de casos específicos. Taylor y Bogdan (1987) definen los conceptos como "...ideas abstractas generalizadas a partir de hechos empíricos." y estos autores disertan sobre el proceso de elaboración de conceptos y lo catalogan de intuitivo, y lo enmarcan dentro de tres pasos prioritarios: primero se busquen palabras y frases del propio vocabulario de los datos recabados que capten el sentido de lo quiere decir el informante, segundo se compare los enunciados y acciones entre sí para ver si existe un concepto que los unifique y tercero a medida que se identifican temas diferentes, buscar las semejanzas y subyacentes que puedan existir entre ellas. Cuando se pueden relacionar los temas de esta manera, hay que buscar una palabra o frase que transmita lo que tienen de similar. El paso final realmente consiste en diseñar un esquema que represente el modelo de autoformación que se pretende estructurar.

\section{Objetivos}

La reflexión autobiográfica permite:

- Conocer y comprender la práctica profesional, realizando un proceso de discriminación de las experiencias docentes, e identificando el grado de significación de las mismas.

- Reflexionar sobre la cotidianidad del aula, buscando la conformación de un ambiente educativo formativo e interrelacionado.

- Facilitar la toma de decisiones en concordancia con los propósitos, medios y contexto educativo. 
- Identificar los puntos claves favorables al desarrollo personal y profesional, con objeto de estructurar un modelo de autodesenvolvimiento profesional.

\section{Metodología}

La metodología utilizada para desarrollar esta autobiografía corresponde a la Clínica introspectiva, se formularon una serie de preguntas, para facilitar el autoanálisis de las experiencias de formación más significativas y con mayor repercusión en el ejercicio profesional. Las preguntas planteadas sirven de guía para la exploración o indagación de la propia acción educativa. Medina (1996) valora lo esencial de la metodología indagadora en la reflexión autobiográfica ya que estimula al docente al esfuerzo de jerarquizar sus experiencias laborales de acuerdo a su incidencia formativa, a justificar sus decisiones pedagógicas y analizar su alcance, así como, profundizar en la interacción de alumnos y docentes, institución y comunidad educativa.

Las preguntas formuladas para la realización de una autobiografía profesional son las siguientes y, tienen propósitos muy específicos, entre ellos se encuentran:

¿Qué experiencias han sido significativas? Esta pregunta conduce a la identificación de personas, asignaturas, trabajo en centros educativos, que de una forma u otra han marcado el ejercicio profesional, también es oportuno la descripción de caracteństicas muy peculiares de las situaciones planteadas.

¿Cuándo y qué duración ha tenido la experiencia? Permite esta pregunta ubicar las actividades más significativas en forma cronológica, e incluso resaltar particularidades del mismo hecho.

¿Cuál es el contexto que ha rodeado la experiencia?. Las experiencias de gran significado formativo, han tenido lugar en un marco concreto, que ha limitado a favorecido el desarrollo profesional.

¿Para qué ha servido esta experiencia formativa? La introspección que se pueda realizar con respecto a una experiencia significativa, implica describir el impacto que ha tenido en la propia vida profesional, buscar la trascendencia de las mismas.

¿Qué dificultades ha encontrado? La identificación de los factores menos favorables al proceso de autoformación profesional, son consideraciones necesarias, cuando se quiere establecer un modelo de autodesenvolvimiento profesional, propiciando un contexto que facilite su aplicación y consolidación.

Para analizar los datos, se organizó la información por preguntas, tratando de sintetizar, las experiencias formativas identificadas, luego se esquematizo la información por pregunta tratando de identificar conceptos que resumieran el sentido de las descripciones realizadas, posteriormente se busco relacionar los conceptos definidos para resumir aún más la información y establecer un modelo de autoformación profesional. El modelo establecido, se diseño tomando como base el grado de significación de vivencias 
profesionales, y su eficiencia es relativa, pues depende de muchos factores que escapan al control e incluso a la introspección realizada, pero es un punto de partida para establecer una reflexión conjunta de autobiografías profesionales y en un espíritu colaborativo, constituir un modelo con más aplicabilidad y efectividad a cualquier grupo de profesionales.

\section{Modelo de autodesenvolvimentofipsional}

Como producto del análisis y reflexión de mi autobiografía profesional, desarrollada en correspondencia con las preguntas orientadoras, se estructura un modelo de autodesenvolvimiento profesional (fig. 1), En este modelo el proceso reflexivo del docente es continuo y su carácter formativo es lo que le imprime a las subsecuentes etapas consideradas una naturaleza experimental y particular en la búsqueda de la mejora y comprensión de una propuesta de autoformación permanente del docente.

El docente identifica las experiencias significativas en el ejercicio profesional, principalmente su contexto, y la resonancia personal que a experimentado en las diversas situaciones relevantes, de tal forma que reconoce aquellas vivencias que han contribuido a su mayor sensibilización como docente, a comprender mejor la realidad y problemática educativa, hecho que le suscita una situación de incertidumbre y lo conduce a la búsqueda de alternativas para dar una respuesta acorde a las circunstancias y generalmente, cuando no es presa fácil del conformismo y la apatía, el docente responde con el diseño de un proceso de autoformación que ya sea o no con propósitos definidos previamente, le estimula a tomar cursos, a realizar estudios sistemáticos (maestría y doctorado), o estudios asistemáticos con objeto de capacitarse y actualizarse, lo que le permite evolucionar en sus creencias docentes,

El tema de las creencias docentes es planteado por diversos autores, entre ellos Porlán (1995) expresa dentro del enfoque constructivista y realiza un planteamiento en el cual admite que los estudiantes y los profesores, poseen creencias y constructos personales elaborados idiosincrásicamente en un marco social determinado. Siendo esto cierto, no basta una conclusión conformista y acrítica en el sentido de afirmar que dichas concepciones espontáneas suelen ser erróneas respecto al conocimiento científico y muy estables y resistentes al cambio. Se trata de dar un paso más hacia la complejidad del fenómeno educativo y de reconocer que también es cierto que los constructos personales son susceptibles de evolucionar significativamente, tal como lo propone el modelo de autodesenvolvimiento profesional, pero todo depende en gran parte, de las características del contexto educativo.

En consecuencia, la evolución de las creencias docentes, lleva consigo un desarrollo personal y profesional estrechamente vinculado, y estimula al docente en su trabajo investigador, le proporciona satisfacción por las metas logradas y le compromete aún más en su labor formadora. 


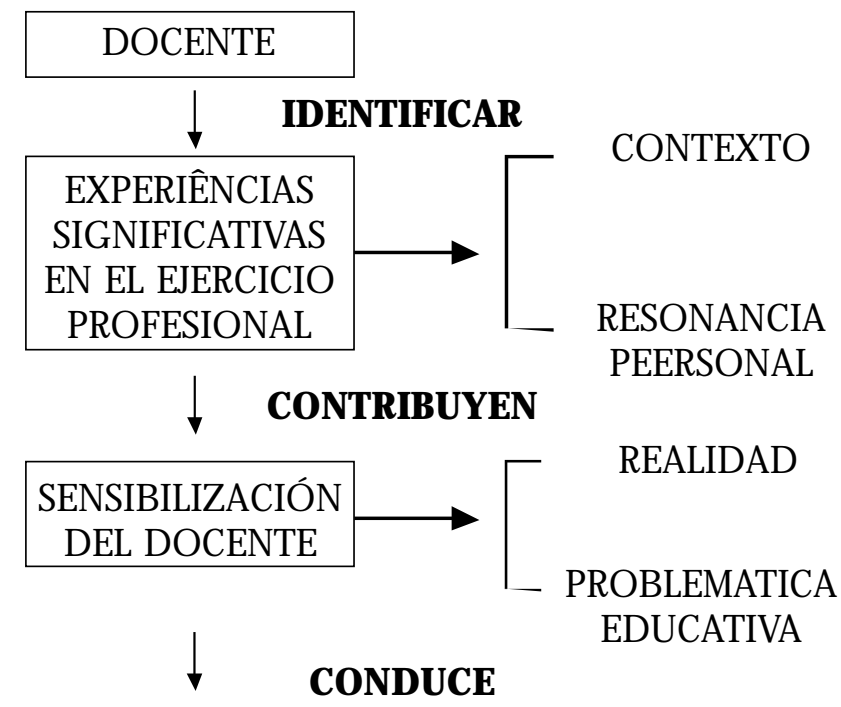

ALTERNATIVAS DE RESPUESTA
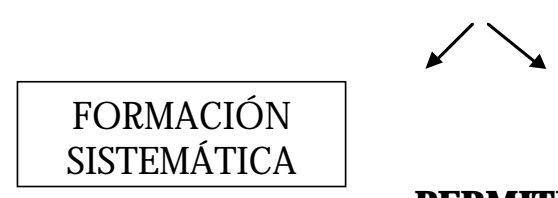
SISTEMÁTICA

FORMACIÓN ASISTEMÁTICA

\section{PERMITE}

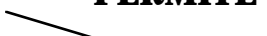

EVOLUCIÓN DE LAS

CREENCIAS DEL DOCENTE

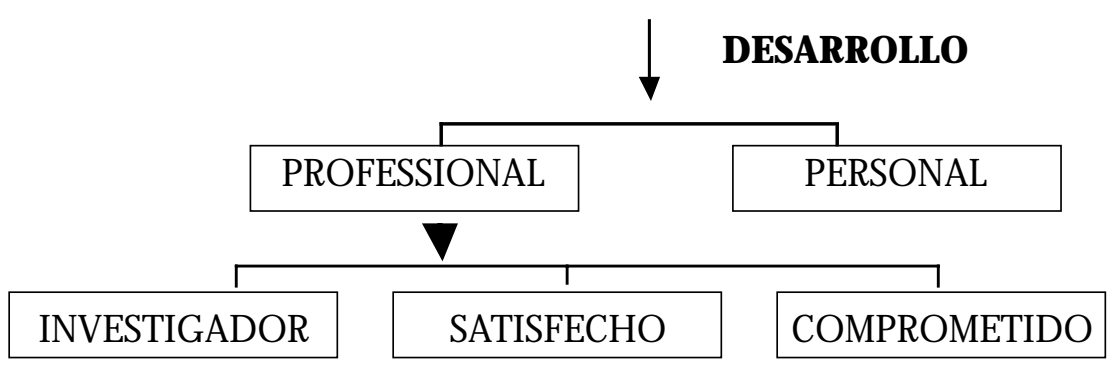




\section{Conclusiones}

- La autobiografía como metodología de investigación y formación del profesorado, incrementa el saber profesional y fundamenta los programas de desarrollo de los docentes y posibilita que cada centro educativo sea un espacio de construcción de cultura innovadora

- Se ha desarrollado una propuesta de un modelo de autodesenvolvimiento profesional, este modelo surge como una determinada forma de interpretar el hecho educativo, y de interrelacionar los elementos pedagógicos, caracterizándolos dentro de un contexto institucional y social. En este caso la interpretación y los principios no responden a una elaboración teórica, sino a la reflexión de experiencias formativas de significado personal, plasmadas en la autobiografía profesional y se llego a la construcción teónica presentada, que pretende interpretar la realidad escolar y dirigirla hacia determinadas metas de desarrollo personal e incluso con trascendencia institucional.

- Se considera el esquema global presentado, como un conjunto de elementos que interactúan unos con otros, de manera que cada componente cobra significado en relación con los demás, formando todos ellos un sistema.

- El modelo propuesto, se realiza con planteamientos sencillos, que motiven al personal docente de un centro educativo a la correflexión de los planteamientos autobiograficos a contextualizarlo y experimentarlo, para lograr retroinformaciones que permitan mejorarlo. Estos esfuerzos sólo adquieren significado cuando son ejecutados y se evidencia su contribución a elevar los niveles de calidad educativa.

\section{Referências Bibliográficas}

DOMINGUEZ,C, MEDINA, A, Y GENTO, S (1997) Autodesenvolvimiento Profesional del Profesorado de. Secundaria. Congreso ECER Franfurt.

MEDINA, A (1988) Didáctica e Interacción en el Aula. Madrid. Cincel.

MEDINA, A (1996) La Autobiografía Modalidad de Formación del Profesorado: Limitacionesy Posibilidades. En E. López-Barajas (coord): Historias de Vida y la Investigación biográfica. Fundamento y Metodología. Madrid. UNED. 95-125.

MEDINA, A., y DOMÍNGUEZ, C (1989) Formación del Profesorado en una Sociedad Tecnológica, Madrid, Cincel.

MEDINA, A, y DOMÍNGUEZ, C (1991) El Empleo del Ordenador en la Enseñanza. Madrid. Cincel.

MEDINA, A, y DOMÍNGUEZ, C (1995) Enseñanza y Curriculum para Personas Adultas. Madrid. EDIPE.

TAYLOR, S, y BOGDAN, R ( 1987) Introducción a los métodos cualitativos de Investigación. Barcelona. PAIDOS.

WOODS, P (1987) La Escuela por Dentro. Barcelona. PAIDOS. 